\title{
IoT Based LPG Gas Level Detection \& Gas Leakage Accident Prevention with Alert System
}

\author{
Zaw Lin Oo, Theint Win Lai and Aung Moe
}

\begin{abstract}
Liquefied Petroleum Gas (LPG) is widely used for cooking fuel in developing countries for economic reasons, for energy-rich fuel source that contains the higher calorific value, for clean fuel with low carbon emission and for a portable that is available in even the faraway of areas. Therefore the proposed gas leakage detection and monitoring system for the gasoline content present in household LPG cylinder is developed. Usually, the capacity of LPG in cylinder is not determined in an exact manner and a cylinder when the gas is about to empty will be a difficult situation for the one who uses LPG gas for cooking continuously. By using IoT, the information of the near to the empty level of LPG gas in the cylinder will send to the user and the gas refill method by using telephonic ordering can be conducted. The purpose of this research is the detection of gas leakage and monitoring the LPG gas cylinder weight regularly to know the remaining value of gas in the cylinder. When the gas leakage is sensed, the warning signal and alarm sound will be active and also switch on exhaust fan automatically to decrease the gas concentration. The weight of LPG is measured using the load sensor (SEN-10245) and the output of the sensor is connected with Arduino MKR Wifi 1010 microcontroller. The user can know the validity of LPG usage daily because the amount of LPG gas will publish as events and watch them come through in real-time using the Wia IoT cloud platform. Consequently, the user is alerted by giving SMS notification to their mobile phone when the LPG level is critically low (below $20 \%$ ) by use of the integration function of the Wia IoT platform call Twilio, using its web service APIs. Then by detecting the gas leakage with MQ6 gas sensor, this research work indicates for gas leakage condition and also helps to prevent the LPG gas burst accidents in the home.
\end{abstract}

Index Terms - LPG Gas Level Detection, Gas Leakage, Arduino MKR Wifi 1010, IoT, Alert System.

\section{INTRODUCTION}

$\mathrm{N}$ OW THE amount of forested area in the nation is dropping and creating environmental issues because the majority of households in Myanmar depend on wood,

ZAW LIN OO, is with Department of Research and Innovation, Division of Atomic Energy, (e-mail: kozawlinoo@gmail.com).

iD https://orcid.org/0000-0001-8132-3263

THEINT WIN LAI, is with Faculty of Computer System and Technology, Info Myanmar University, (e-mail: t-winlei@imu.edu.mm).

iD https://orcid.org/0000-0002-4148-5812

AUNG MOE, is with Department of Research and Innovation, Division of Atomic Energy, (e-mail: aung2011m@gmail.com).

iD https://orcid.org/0000-0003-0846-2704

Manuscript received June 2, 2021; accepted October 25, 2021.

DOI: $\underline{10.17694 / \mathrm{bajece} .946789}$ charcoal, and electricity. LPG is one of the by products resulting from the refining of petroleum, being predominantly composed of the mixture of two hydrocarbons, Propane (C3H8) and Butane (C4H10). When held under pressure, or cooler conditions, it transforms into a liquid state, and is relatively stable. This facilitates their storage and useful in domestic sectors for cooking. So, LPG could play a vital role in Myanmar, helping the environment and excellent substitute for firewood and charcoal. The availability and accessibility of LPG are now increasing as ordinary usage across Myanmar in place of firewood and charcoal for cooking. LPG (either Butane or Propane), is a colorless odorless liquid and contains in the cylinder on the highly concentrated state.

Gas leakage is the major problem for LPG gas burst accidents and People can't know that the gas is leaking. So, several research works and projects have been implemented for gas leakage detection. The existing research provides a gas leakage accident prevention system with an alert which is mainly meant to detect Gas leakage in the kitchen and was proposed with only on-site alarm.

The main objective of this research is to the continuous measurement of the weight of the cooking gas cylinder but in addition, can store this information over time to the IoT platform and how much amount of gas is spent in a week or a month. When the weight reaches the minimum threshold it will automatically sends an SMS alert to the housewife to chain or refill LPG gas cylinder for regular cooking. This system also designed to detect and sense for liquid petroleum gas (LPG) leakage and the alarm unit will be activated immediately, if the amount of gas concentration exceeds normal level to prevent accidents in the kitchen environment.

There have been reviewed several types of research and projects related to the LPG gas monitoring and gas leakage detection system. SmartGas: A smart platform for cooking gas monitoring [1] used a mobile application to detect the amount of LPG gas remaining in a gas bottle. Arduino UNO R3 microcontroller board with an Ethernet shield is used to obtain the information from the sensors and send data to MySQL local database. The mobile application was developed to access the information from the database server and will provide information of LPG gas consumption.

Automated unified trolley system for LPG leakage detection with safety measures and refill booking [2] proposed a system that uses PIC 16F877a for LPG leakage detection and automatic gas booking system. This system gets alerted through the message on gas leakage detection and the cylinder gets automatically booked to the agency when it going to 
empty using GSM SIM 800/300/900 module. Easily available and reliable IoT technology is not used in this research and gas distribution companies need user registration and database system for each gas cylinder with a corresponding phone number.

A wireless gas leakage \& level detection with auto-renewal system [3] detect gas leakage and continuous monitoring of the level of the LPG gas at the time of dispatch and throughout the usage. GSM/GPRS wireless modem sends SMS alert using ARM7 microcontroller when the solid-state gas sensor MQ-5 sense gas leak. The main intention of this research work is the household safety system and gas leakage prevention. IoT technology is not used and an automatic accident prevention system is not conducted in this unit.

Arduino based LPG gas monitoring and automatic cylinder booking with alert system [4] proposed gas leakage detection system with alert message by buzzing the buzzer and trough SMS to the house holders. This system also includes continuous LPG gas detection and automatic cylinder booking with alert system. Arduino Mega2560 micro controller is used with MQ-4 gas sensor and LM35 temperature sensor to prevent major accidents. Load cell is used to continuously measure the weight of the cylinder and automatic rebooking of the new gas cylinder will conduct using SIM800 quad-band GSM / GPRS device to the mobile phone interface. But, fire accident prevention system for gas leakage detection is not considered in this system.

\section{DESIGN AND TECHNIQUES}

This paper will solve the problem for not only detects any leakage of the LPG gas but removes automatically the leaked gas to prevent from fire accidents and it also alerts the user by creating alarm song and warning signal. This proposed method consists of gas leakage detection system, gas cylinder weight measurement module, alert system, microcontroller, IoT system for gas consumption record and cloud communications platform for SMS.

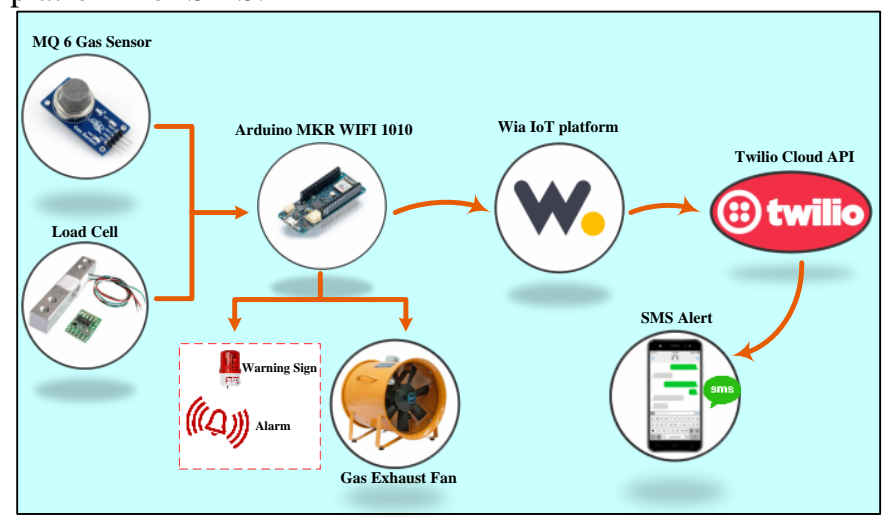

Fig.1. Block Diagram of Proposed System

\section{A. Arduino MKR WiFi 1010}

MKR WIFI 1010 is the evolution of the MKR1000 and is equipped with an ESP32 module made by U-BLOX. The MKR Wifi 1010 aims to speed up and simplify the prototyping of WI-FI based IoT applications thanks to the flexibility of the ESP32 module and its low power consumption. The design includes a Li-Po charging circuit that allows the Arduino MKR WIFI 1010 to run on battery power or external $5 \mathrm{~V}$, charging the Li-Po battery while running on external power. Switching from one source to the other is done automatically. A good 32 bit computational power, the usual rich set of I/O interfaces, low power Wi-Fi with a Cryptochip for secure communication, and the ease of use of the Arduino Software (IDE) for code development and programming. All these features make this board the preferred choice for the emerging IoT battery-powered projects in a compact form factor. The USB port can be used to supply power (5V) to the board. The Arduino MKR WIFI 1010 is able to run with or without the Li-Po battery connected and has limited power consumption [5].

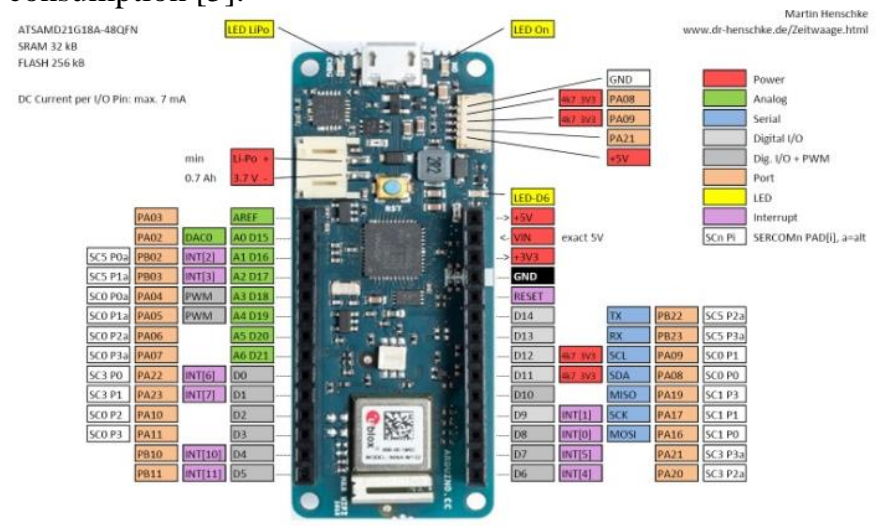

Fig.2. Arduino MKR Wifi 1010 Board

https://www.henschke-geraetebau.de/dr/MKR_WiFi_1010_Pinout.pdf

\section{B. MQ-6 gas sensor}

MQ-6 is a Sensor for Natural Gases Sensitive material. MQ-6 gas sensor is $\mathrm{SnO}$, which has lower conductivity in clean air. When the target combined gas exists, the sensor's conductivity is heavier with the gas concentration rising. The simple electro circuit is used to convert the change of conductivity to gas concentration level. MQ-6 gas sensor has high sensitivity to LPG, Methane, Propane, Butane, and also response to Natural gas. The sensor can be used to detect different combustible gas, especially Methane; it is costeffective and useful for various applications. MQ-6 gas sensor is composed of micro AL2O3 ceramic tube, Tin Dioxide $(\mathrm{SnO} 2)$ sensitive layer, measuring electrode and heater are fixed into a crust made by plastic and stainless steel net. The heater provides necessary work conditions for the work of sensitive components. The enveloped MQ- 6 has 6 pins, 4 of them are used to fetch signals, and the other 2 are used for providing heating current [6].

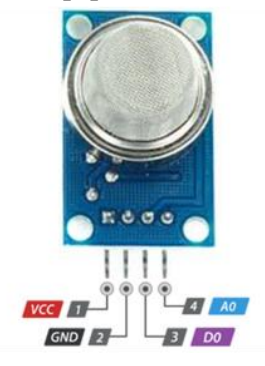

Fig.3. MQ-6 Gas Sensor 


\section{Load sensor}

A load cell is a passive transducer or sensor that measures force and outputs this force as an electrical signal. The load cells are used since it provides an accurate weight. Straingauge is used in most of the load cells for accurate measurement. The gauges are bonded onto a beam or structural member that deforms when weight is applied, in turn deforming the strain-gauge. As the strain gauge is deformed, it's electrical resistance changes in proportion to the load. It is often easy to measure the parameters like length, displacement, weight, etc that can be felt easily by some senses. However, it is very difficult to measure the dimensions like force, stress, and strain that cannot be really sensed directly by any instrument. This is used to measure the weight of the LPG gas cylinder. The accuracy rate is less than $0.1 \%$ of the full scale [7].

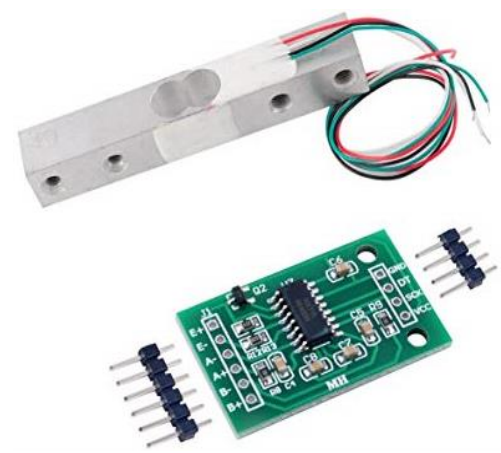

Fig.4. Load Cell 1 kg weight sensor and HX711 amplifier weighing module

\section{Wai IoT}

Wia is a cloud platform that aims to provide developers with a scalable and powerful backend for their Internet of Things (IoT) solutions. All of Wia's features are either available through a REST API or a web-based dashboard. Moreover, Wia also offers a very lightweight messaging protocol (MQTT) to the developers who want to stream events and logs. For using the minimum of the data, Wia has also designed for communication between clients and the backend. Wia platform supports the open-source libraries like Node.JS and $\mathrm{iOS}$ and other most popular web technologies. Wia is the only platform for real-time IoT applications and can add any development board to create projects around the platform. Wia can easily communicate with other third-party tools using integration services to send notifications, store data in cloud storage, perform image recognition and lots more abilities. Mobile and web apps can build to interact with IoT devices from anywhere in the world [8].

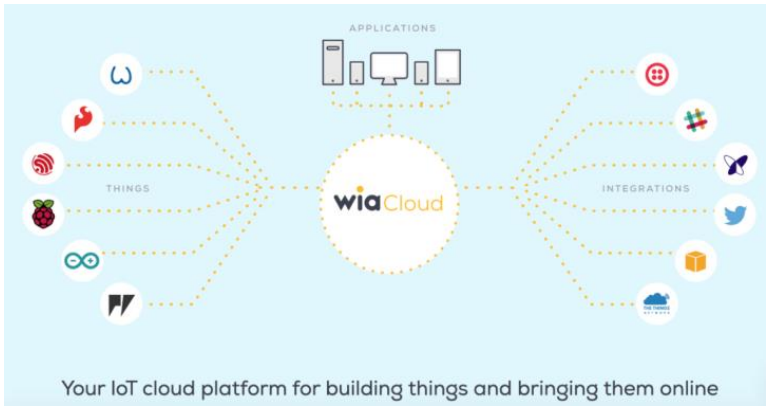

Fig.5. Wai IoT platform

\section{E. Twilio}

Twilio is a cloud communications platform that can send and receive global SMS, MMS, and IP messages from any app, with a simple and powerful API. Twilio is a service that gives developers possibility to programmatically make and receive phone calls and send and receive text messages using its web service APIs. Twilio's services are accessed over HTTP and are billed based on usage.

In fact, Twilio powers communications for over 40,000 businesses around the world. Twilio is a developer platform for communications. Software teams use Twilio APIs to add capabilities like voice, video, and messaging to their applications. This enables businesses to provide the right communications experience for their customers. Behind Twilio APIs is a Super Network, a software layer that connects and optimizes communications networks around the world. This is what allows the users to reliably call and message anyone anywhere [9]. Twilio has taken the global telecom network and turned it into a cloud communications platform with these capabilities and more [9].

Voice: API and SDKs to build calling capabilities within web and mobile apps. Connect to landlines, mobile devices or even WebRTC clients to make calls from apps or power multinational call centers [9].

Video: Real-time video infrastructure and SDKs to embed video collaboration and context-sharing into your web or mobile app. A global infrastructure that handles signaling, registration, and media relay [9].

Messaging: API and SDKs to send and receive SMS, MMS, and IP messages globally from your web and mobile app, and use intelligent delivery features to ensure messages get through [9].

Authentication: Two-factor authentication service to strengthen and even replace traditional username and password login for websites, SaaS products, and mobile apps.

Connectivity: Global carrier connectivity services made simple and available instantly with no upfront contracts. Local and toll-free phone numbers around the globe along with SIP connectivity made available as APIs.

Twilio are used by More than a million developers and leading brands to build innovative communications solutions. At Coca-Cola Enterprises, field technicians receive automated alerts as soon as vending machines need maintenance and also Lyft and Match.com enable users to communicate directly with each other without revealing their private phone numbers [9].

In this project, Programmable connectivity options of Twilio provide virtual phone numbers and messaging connectivity with devices are used.

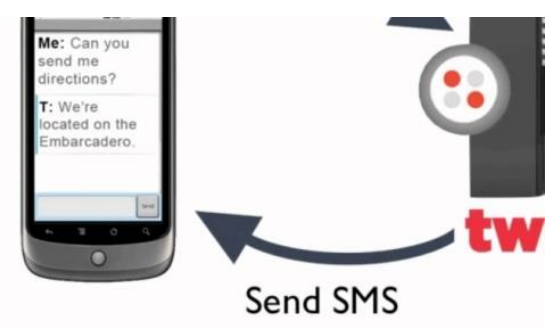

Fig.6. Twilio SMS API 


\section{IMPLEMENTATION OF SYSTEM}

Load cell with HX711 amplifier weighing module and MQ6 gas sensor are used as input devices for gas leak and level detection system. MQ-6 gas sensor is provided for gas leakage detection system. Load cell and amplifier unit used as a gas cylinder weight measurement module. Alert system included warning signal and alarm sound for gas leakage detection and SMS alert for gas cylinder refilling. For gas burst accident prevention system, the gas concentration is decreased by turning on the exhaust fan. The level of LPG gas in cylinder measured and send to Wia IoT cloud. Twilio cloud API will send an SMS alert automatically to the consumer when the gas level reaches the minimum threshold value using the thirdparty tools of Wia.

In this research, HX711 amplifier module contains 24-bit high-precision analog-to-digital converter chip to interface directly with a load sensor. This chip has two analog input channels and designed for high-precision electronic scale applications. Either Channel A or B differential input can be selected to the low-noise programmable gain amplifier (PGA). Channel A can be programmed with a gain of 128 or 64-bit and Channel B has a fixed gain of 32-bit. Onchip power supply regulator eliminates the need for an external supply regulator to provide analog power for the ADC and the sensor [11].

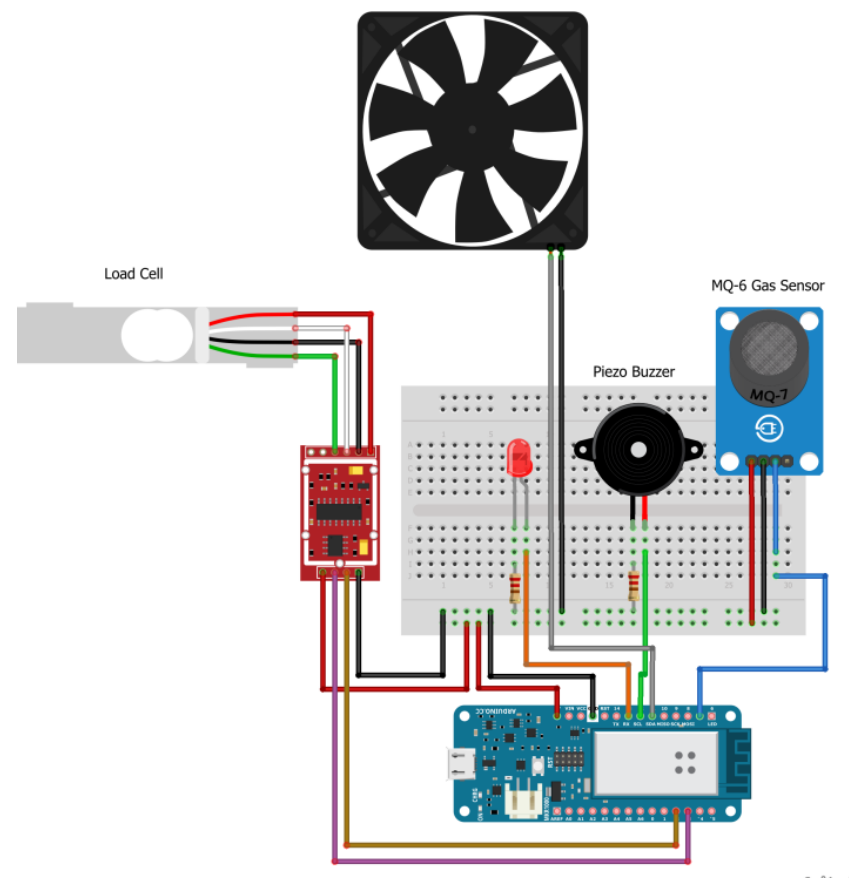

Fig.7. Circuit diagram for gas leak and level detection system

Red, black, green and white wire of load cell connect to E+, E-, A- and A+ port of HX711 board respectively. The HX711 amplifier unit pin (SCK) was attached to D2 and pin (DT) connected to D3 of Arduino MKR WiFi 1010. The Operation supply voltage of the amplifier unit is $4.8-5.5 \mathrm{~V}$. So, the Vcc pin of the unit connected to the Arduino $5 \mathrm{~V}$ power pin. MQ-6 gas sensor is an electro-chemical type sensor. The output digital signal from that sensor is (D out) pins connect to Arduino (D7) pin. The sensitivity range of the sensor can be adjusted using built-in potentiometer. Vcc pin of the sensor was connected to the Arduino 5V pin as MQ6 gas sensor operates on 5V DC and attaches GND pin to the ground pin on the Arduino. Pin D12, D13, and D11 are connected with piezo buzzer, warning led and gas exhaust fan respectively. An Arduino library of $\mathrm{hx} 711$ is required for weight scales measurement of this project. All the hardware components are developed as a circuit diagram and that shown in Fig. 7.

\section{RESULTS AND DISCUSSION}

The hardware implementation of the project has classified into two parts. Designing of the gas leakage detection and gas level measurement are included. This project is designed for the kitchen that used LPG for cooking. For a gas leakage detection unit, the MQ-6 gas sensor is connected with Arduino MKR WiFi 1010. The alarm unit will activate and also turn on the exhaust fan automatically when LPG concentrations are over $500 \mathrm{ppm}$ in the kitchen.

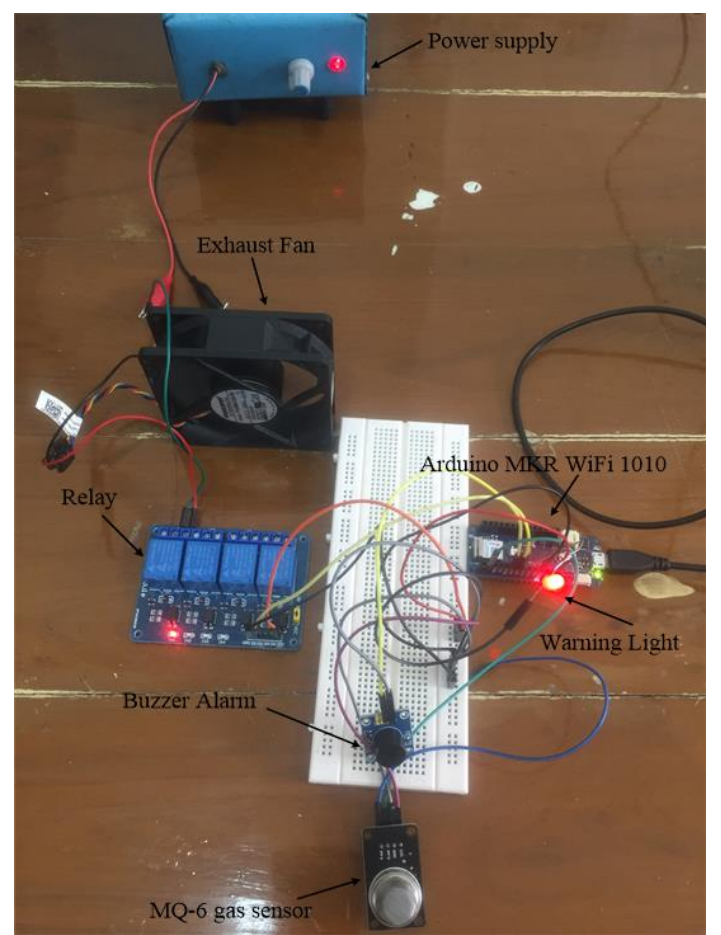

Fig.8. Gas leakage detection and Alert system testing

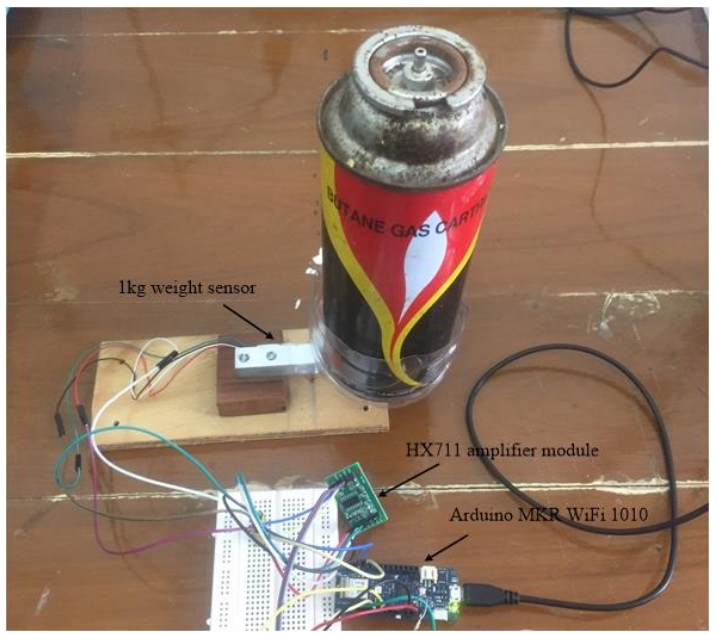

Fig.9. Gas level measurement system testing 


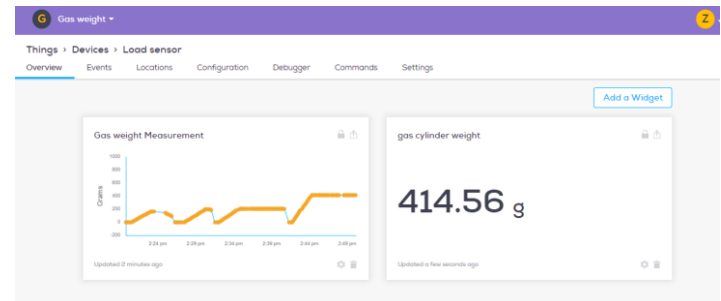

Fig.10. LPG gas level in Wia IoT cloud Platform

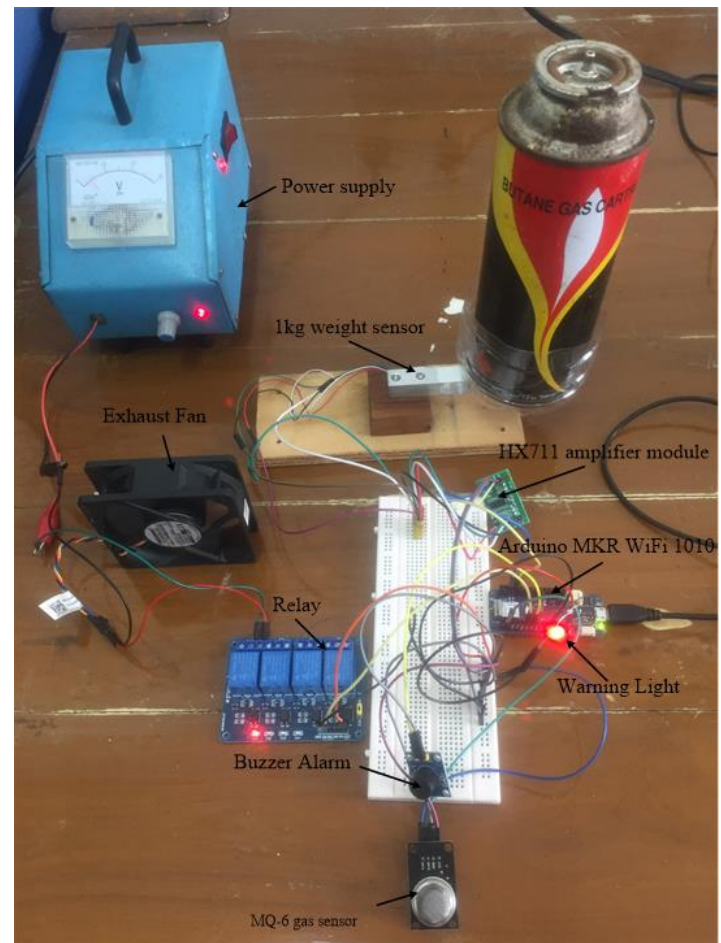

Fig.11. Prototype of LPG Gas Level Detection \& Gas Leakage Accident Prevention with Alert System

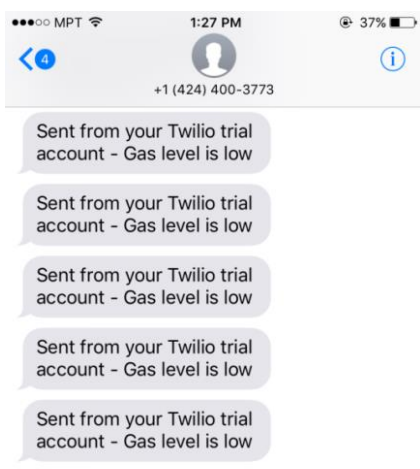

(i) $(1)$

Sent from your Twilio trial ccount - Gas level is low Sent from your Twilio trial ccount - Gas level is low

Sent from your Twilio trial account - Gas level is low

Sent from your Twilio trial Sent from your Twilio trial account - Gas level is low of LPG gas. The Twilio cloud API will send an SMS alert to the housewife when the LPG level is critically low (below $20 \%$ ) to chain or refill the LPG gas cylinder.

\section{CONCLUSION}

This paper is focused on the gas leakage detection system for home safety and will update regularly about LPG consumed daily. The developed system is affordable cost as components used here are cheap when compared to gas detectors commercially available in the market. LPG leakage is alerted by activating the alarm and using an exhaust fan it removes the leaked gas from the area. LPG consumption is observed and can be booked for a new LPG cylinder by the customer when the gas level is near empty. In the future, the gas pressure sensor module can be used to detect highpressure gas in a cylinder pipe, displaying the alert messages via SMS and LCD displays.

\section{REFERENCES}

[1] Gabriel V. da Silva Medeiros, Matheus Ricardo dos Santos, Alba Sandyra Bezerra Lopes, Edmilson C. Barbalho Neto "SmartGas: A smart platform for cooking gas monitoring". 2017 IEEE First Summer School on Smart Cities, Natal, Brazil, August 6-11, 2017, pp. 97-102.

[2] Shraddha Suresh Tanksale, Prof. A.S. Mali and Dr. B.T. Salokhe, "Automated Unified Trolley System for LPG Leakage Detection with Safety Measures and Refill Booking". International Journal of Engineering and Management Research, Volume-8, Issue-3, June 2018, pp: 224-228.

[3] S. Sivajothi Kavitha, S. Senthilkumar, "A Wireless Gas Leakage \& Level Detection with Auto Renewal System". International Journal of Advanced Research in Electrical, Electronics and Instrumentation Engineering, Vol. 4, Issue 4, April 2015, pp: 2095-2100.

[4] R. Naresh Naik, P. Siva Nagendra Reddy, S. Nanda Kishore, K. Tharun Kumar Reddy, "Arduino Based LPG gas Monitoring \& Automatic Cylinder booking with Alert System". IOSR Journal of Electronics and Communication Engineering (IOSR-JECE), Volume 11, Issue 4, Ver. I (Jul.-Aug .2016), PP 06-12.

[5] Zaw Lin Oo, Theint Win Lai, Aung Moe, "Iot Based Low-cost Physical Protection and Alarm System for Gamma Irradiation Facility". Conference on Science and Technology Development (CSTD-2019), Oct 31- Nov 1, 2019.

[6] "MQ-2 Semiconductor Sensor for Combustible Gas", [online]. Available: https://www.pololu.com/file/0J309/MQ2.pdf.

[7] "YZC-131A Load Cells datasheet" [online]. Available: https://www.electronicoscaldas.com/datasheet/YZC-131A.pdf .

[8] "Wia Provides Cloud Infrastructure for IoT" [online]. Available: https://www.infoq.com/news/2016/01/wia-iot-cloud-platform/.

[9] "What is Twilio and How Do Twilio APIs Work?" - Twilio 101 Twilio [online]. Available: https://www.twilio.com/learn/twilio101/what-is-twilio.

[10] "What Can You Do with Twilio?" [online]. Available: https://www.twilio.com/learn/twilio-101/what-can-you-do-with-twilio

[11] "HX711 Load Cell Amplifier Interface with Arduino" [online]. Available: https://circuits4you.com/2016/11/25/hx711-arduino-loadcell/

\section{BIOGRAPHIES}

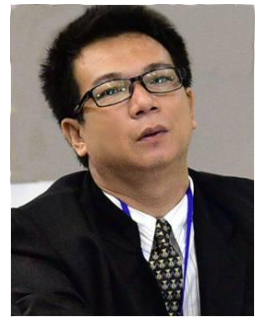

ZAW LIN OO was born in Yangon, Myanmar, in 1979. He received B.E (Bachelor of Engineering) and M.E (Master of Engineering) degrees in Nuclear Engineering from Yangon Technological University, Yangon, (Myanmar) Burma, in 1999 and 2003, respectively and a Ph.D. degree in 
Nuclear Engineering in the year 2007 from Yangon Technological University, Yangon, (Myanmar) Burma. His research interests include computer science and engineeringembedded systems, cloud computing, artificial intelligence, Electrical and electronic engineering- electronic, control system.

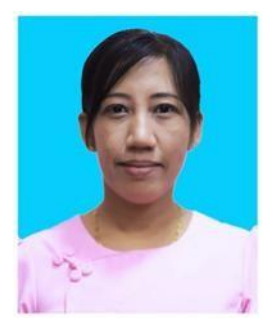

THEINT WIN LAI is an associate professor at the Faculty of Computer System and Technology, Info Myanmar University, Yangon, (Myanmar) Burma. She received the B.S. degrees in Physics from the Pathein University, in 1999 and the M.S. degree in computer Hardware Technology from Yangon Technological University, Yangon, (Myanmar) Burma, in 2002. She received the Ph.D. degree in computer Hardware Technology from University of Computer Studies, Yangon (UCSY), in 2006. Her research interests are computer science and engineering- embedded systems, cloud computing, artificial intelligence, Electrical, electronic engineering- electronic, control system and IoT based control system.

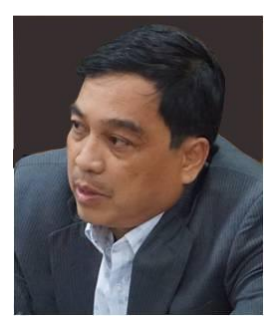

AUNG MOE is a Director in the Myanma Scientific and Technological Research Department at the Ministry of Science and Technology. Nay Pyi Taw, Myanmar (Burma). He received B.Sc and M.Sc degrees in Physics from University of Yangon, Yangon, (Myanmar) Burma, in 1992 and 1997, respectively. He received the Ph.D. degree in Nuclear Engineering from National Research Nuclear University MEPhI (Moscow Engineering Physics Institute), in 2006. His research interests are computer science and engineering- embedded systems, Advanced diagnostic systems, and Advanced Information System Modeling Technologies. 